\title{
La lutte pour la reconnaissance des droits des femmes dans le contexte révolutionnaire russe
}

\section{Thomas Franck}

\section{OpenEdition \\ Journals}

Electronic version

URL: https://journals.openedition.org/grm/2608

DOl: $10.4000 / \mathrm{grm} .2608$

ISSN: $1775-3902$

\section{Publisher}

Groupe de Recherches Matérialistes

\section{Electronic reference}

Thomas Franck, "La lutte pour la reconnaissance des droits des femmes dans le contexte révolutionnaire russe", Cahiers du GRM [Online], 17 | 2020, Online since 02 January 2021, connection on 29 September 2021. URL: http://journals.openedition.org/grm/2608; DOI: https://doi.org/10.4000/ grm.2608

This text was automatically generated on 29 September 2021.

(c) GRM - Association 


\title{
La lutte pour la reconnaissance des droits des femmes dans le contexte révolutionnaire russe
}

\author{
Thomas Franck
}

1 Le rôle joué par les femmes dans le processus révolutionnaire initié au début du $\mathrm{XX}^{\mathrm{e}}$ siècle en Russie est capital et reste encore assez peu reconnu ${ }^{1}$. Le soulèvement insurrectionnel des ouvrières, des étudiantes et des employées en février 1917, la présence non négligeable d'intellectuelles dans les rangs sociaux-démocrates, socialistes-révolutionnaires et anarchistes ainsi que les revendications en faveur d'une reconnaissance des droits des femmes illustrent la propension à une convergence des luttes. Le renversement d'un modèle sociétal féodal et d'une économie marquée par des inégalités et des privilèges importants (ceux d'un patriarcat structuré autour d'une monarchie religieuse et ceux de l'exploitation industrielle) se couple à un projet de transformation des institutions morales et sexuelles défendues sous les Tsars.

2 Toutefois, si les premiers moments de la révolution bolchevique-soviétique sont marqués par une reconnaissance de l'égalité formelle entre les hommes et les femmes et par le (relatif) accès de celles-ci à la sphère politique, le tournant conservateur et nataliste insufflé sous Staline dans le courant des années 1930 constitue un reniement de ces idéaux révolutionnaires. Un véritable aller-retour s'opère du régime patriarcal des Tsars à la domination masculine propre au stalinisme, où la figure du père des peuples est dominante. Deux exemples sont à ce titre emblématiques : l'avortement et le divorce. Dépénalisés sous Lénine, ils deviennent progressivement marginalisés par décrets sous Staline en 1936 puis en 1944. Pour bien comprendre l'histoire de la révolution soviétique sous l'angle des luttes pour la reconnaissance des droits des femmes, il est fondamental de penser la relation entre la structure de la famille, plus largement de l'intimité, et la structure économique, celle des rapports de production. En effet, la famille et la place conférée aux femmes dans le système autocratique et patriarcal des Tsars reflètent le statut de propriété d'un père ou d'un mari à laquelle celles-ci sont réduites. La désintégration quasi complète des codes et des normes qui 
fondent cette domination au cours des événements successifs à la révolution traduit quant à elle le moment d'une modification profonde des structures sociales, politiques et économiques. Enfin, le moment stalinien réintroduit dans la société soviétique une domination - par l'exploitation et par la répression de toutes les couches sociales, y compris les plus pauvres - qui se solde notamment par une remise en cause de l'autonomie politique des femmes.

3 La thèse et les lectures développées par Fabio Bruschi à la suite d'Althusser à propos du matérialisme politique de la rencontre éclairent les conditions du basculement d'un régime à l'autre et la convergence des luttes politiques des femmes au moment de la révolution russe. La destruction du régime patriarcal des Tsars couplée au contexte de guerre, qui amène les femmes à occuper une place de plus en plus importante dans l'industrie et la gestion des campagnes, ainsi que la présence importante de femmes dans les rangs révolutionnaires et syndicaux constituent des facteurs politiques déterminants dans cette histoire révolutionnaire. En outre, l'émergence de plusieurs mouvements insurrectionnels internationaux plus ou moins spontanés en réaction à une guerre jugée impérialiste et classiste rencontre, en Russie, des milieux révolutionnaires professionnels organisés de longue date, souvent dans la clandestinité, et conscients de la nécessité d'une mise à mal des institutions morales contraignant l'émancipation du prolétariat féminin. Son exploitation dans des modes d'organisation de plus en plus marqués par une confiscation des outils de production et de la plus-value salariale donne naissance à une progressive ouverture au référentiel socialiste. La clandestinité et l'agitation politique au sein des comités ouvriers sont également des facteurs clefs dans la préparation d'une radicalité politique, qui sera portée par de nombreuses révolutionnaires au sein et en marge des partis socialisterévolutionnaire, menchevique et bolchevique.

\section{Celles qui ont fait la révolution}

Nadejda Kroupskaïa, Alexandra Kollontaï et Catherine Breshkovsky représentent l'échiquier politique révolutionnaire au moment de la révolution de 1917. Si les deux premières sont proches du bolchevisme - avec davantage d'hétérodoxie pour la seconde, dans un premier temps menchevik -, la troisième est membre d'une mouvance extérieure à la social-démocratie russe, les socialistes-révolutionnaires. Héritiers du populisme russe, dont Marx était idéologiquement proche, les SR sont composés depuis le XIX ${ }^{e}$ siècle d'un nombre non négligeable de femmes. En défendant l'alphabétisation massive des couches populaires, la gratuité des soins et de l'enseignement ou encore l'union libre, Kroupskaïa, Kollontaï et Breshkovsky mobilisent des conceptions assez proches de l'action politique, bien qu'elles ne puissent nullement être réduites à un programme commun. La diversité idéologique est l'une des caractéristiques des années périphériques à la révolution. Mais, ce que visent communément ces revendications est une émancipation sociale et morale des femmes, une libération des contraintes subies quotidiennement dans les régimes féodaux mais aussi bourgeois. Déjà Engels faisait appel, dans son Origine de la famille, de la propriété privée et de l'État, à la nécessité d'une absence d'ingérence de l'État dans les affaires privées $^{3}$. On rappellera à ce propos la célèbre maxime de Kollontaï défendant «Une union libre de libres individus »: "Sur les ruines de l'ancienne famille nous verrons bientôt s'en élever une nouvelle qui développera des rapports entièrement différents 
entre homme et femme et qui sera une union d'affection et de camaraderie, l'union de deux individus égaux de la société communiste, tous deux libres, tous deux indépendants, tous deux travailleurs $»^{4}$. Dans La base sociale de la question féminine, Kollontaï défend l'idée d'une "femme nouvelle », libérée de l'oppression subie dans le modèle bourgeois dominant :

N'avons-nous pas découvert finalement l'aspect de la question féminine qui unit les femmes de toutes les classes? Ne pouvons-nous pas lutter ensemble contre les conditions qui les oppriment? N'est-il pas possible que les souffrances, les revendications que les femmes partagent dans ce domaine n'atténuent les antagonismes de classe et n'offrent des aspirations communes, la base d'une action conjointe pour les femmes de différents camps? Les féministes luttent pour des formes plus libres du mariage et pour le "droit à la maternité »; elles élèvent leur voix pour défendre les prostituées, l'être humain persécuté par tous. Voyez combien la littérature féministe est emplie de la recherche de nouvelles formes de relations et de demandes enthousiastes pour l'« égalité morale » des sexes. N'est-il pas vrai que, tandis que dans la sphère économique la bourgeoisie est à la traîne de l'armée de millions de femmes prolétaires, c'est elle au contraire qui ouvre la voie de la «femme nouvelle ", dans une résolution de la question familiale. ${ }^{5}$

Cette lecture révèle que ce sont des facteurs économiques qui soutiennent et renforcent la subordination des femmes dans un mode de production fondé sur la division du travail et sur son organisation hiérarchisée, quelle que soit la classe de ces femmes (prolétariat, bourgeoisie ou Lumpenproletariat). L'égalité réelle ne peut dès lors être pensée que sur base d'une transformation profonde et radicale des structures économiques et sociales, au fondement des transformations morales. L'exploitation du travail par le capital et l'aliénation à une structure féodale patriarcale (soutenue par plusieurs institutions, dont la Cour des Tsars, la Douma, l'Église Orthodoxe et l'okhrana) sont les origines mêmes de la révolution émancipatrice.

Dans l'héritage de Marx et d'Engels, Rosa Luxembourg, communiste allemande assassinée en 1919 par une social-démocratie réformiste, avait développé plusieurs réflexions sur les relations entre les luttes d'émancipation collective et les luttes pour la liberté individuelle des femmes. Son texte Suffrage féminin et lutte de classes, qui mentionne l'important journal de Clara Zetkin L'Égalité, insiste sur la nécessité d'une conscientisation politique au sein du prolétariat féminin ${ }^{6}$. Luxembourg, qui n'a pas hésité à émettre de dures critiques à l'encontre du léninisme, était opposée au centralisme du parti des bolcheviks et préférait défendre une conception insurrectionnelle propre à la spontanéité révolutionnaire des masses. Cette spontanéité ne doit nullement être comprise comme une valorisation romantique du prolétariat prétendument conscient de son rôle révolutionnaire. Au contraire, Luxembourg considère que le travail politique et idéologique de l'avant-garde révolutionnaire est d'amener la classe ouvrière, dans une logique de collaboration et d'autonomie, à la pleine conscience de soi. Il ne sert selon elle à rien de placer quelques intellectuels ou intellectuelles au pouvoir si la masse n'a pas consciemment intégré le processus politique, si elle n'a pas développé une " nouvelle culture " propre - au sens gramscien ${ }^{7}$ - et si elle n'a pas organisé une auto-éducation populaire de grande ampleur. Et le rôle $\mathrm{du}$ prolétariat féminin est en ce sens capital pour la militante, qui considère son action comme la possibilité d'une émancipation collective intégrale. Elle ne peut en outre admettre de lutte pour la reconnaissance des droits des femmes sans l'action directe de celles-ci, sans une participation massive aux organisations et aux décisions sociales et 
politiques, sans une maîtrise des outils de production et des institutions économiques, juridiques et morales.

7 Dans la section 65 du Cahier 7 intitulée "Féminisme ", Antonio Gramsci insiste à ce propos sur la manière dont la mise à mal d'une double domination (domination exercée sur le prolétariat et domination exercée sur la femme) répond à l'injonction d'une émancipation de tous : «tout mouvement historique qui innove n'est mûr que dans la mesure où y participent non seulement les vieux, mais aussi les jeunes, les personnes d'âge mûr et les femmes, de telle sorte qu'il a une influence même dans l'enfance $»^{8}$. Voici formulée, avec Gramsci et Luxembourg, une conception divergente du dirigisme centralisé bolchevique des années d'après-révolution. Cette logique centralisée et dirigiste résulte d'un contexte de clandestinité et de difficultés liées à l'illégalité instaurée par les Tsars depuis des décennies'. Elle s'accentue à la suite de la radicalisation oppositionnelle des violences durant la Guerre civile (1918-1921). Les querelles entre les fractions bolchevique et menchevique (elles-mêmes parcourues de tensions internes), qui les ont clivées de 1903 à 1912, sont complexes et ne permettent pas d'opposer de façon duale deux «blocs » politiques et idéologiques. Chacun défend, au gré des évolutions historiques, tantôt des conceptions dirigistes et excluantes, tantôt des tentatives d'ouverture, entre inclusion, débat interne et clandestinité. La progressive instauration d'un dirigisme centralisé et d'une conception sectaire du parti dans le courant des années 1920 résulte donc d'une conjoncture bien précise, celle qui a obligé les groupes militants à définir leur action politique dans la clandestinité, dans une illégalité, et qui les a contraints à une réaction violente et rigoureusement organisée dans un contexte criminogène, celui des répressions meurtrières des manifestations, des attentats politiques, de la Guerre mondiale et de la Guerre civile ce contexte criminogène est d'ailleurs accentué par la terreur de la contre-révolution blanche. Le fonctionnement de l'avant-garde dirigeante et centralisée des bolcheviks, dont l'autorité politique va s'affirmer aux dépens de l'auto-éducation du prolétariat dans sa globalité, n'est dès lors pas inscrite dans le programme idéologique originel des bolcheviks. Pierre Broué insiste bien sur le fait qu'il n'y a pas de rapport causal direct entre l'appareil du parti clandestin et l'appareil du parti au pouvoir mais bien une transformation conjoncturelle, Lénine lui-même ayant défendu contre Rykov l'inclusion des ouvriers dans les comités ${ }^{10}$. La faiblesse quantitative des expériences ouvrières en Russie, dans une structure économique majoritairement rurale, ainsi que la volonté de substituer le parti à une classe trop peu nombreuse amènent Lénine à privilégier une hétéronomie décisionnelle à l'autonomie politique préconisée dans plusieurs œuvres du communisme philosophique. Celles-ci se sont majoritairement construites, dans les pays de l'Europe occidentale, sur base d'expériences ouvrières déjà nombreuses, parfois expérimentales et pour certaines fondées sur une réelle conscience de classe organisée : l'Angleterre et la Prusse pour Marx, l'Allemagne pour Luxembourg, l'Italie pour Gramsci. Ces remarques visent à comprendre la manière dont le prolétariat féminin russe, à l'origine déterminant dans le processus révolutionnaire, a été progressivement marginalisé par l'organisation du parti bolchevique, lui-même contraint dans son fonctionnement et sa structure par des conjonctures diverses. La reproduction d'un modèle politique vertical et hiérarchisé reconduit plusieurs dominations et laisse difficilement la place à une visibilité du prolétariat féminin.

8 Avant la prise de pouvoir bolchevique, c'est une véritable conscience politique, couplée à une lutte pour la reconnaissance des droits syndicaux et de réunion, qui est à l'origine des mouvements de février 1917, moment qui s'inscrit dans l'histoire longue et 
continue des luttes du mouvement ouvrier féminin. Revendiquant du pain et une interruption de la guerre, les ouvrières de Petrograd défilent dans les rues, perpétuant ainsi une tradition très ancrée de la grève dans les usines du textile russes au sein desquelles les femmes sont nombreuses, voire majoritaires - on se souviendra de la célèbre grève d'Orekhovo-Zouïevo qui aboutit, en 1885, à l'interdiction du travail de nuit des femmes et des jeunes.

\section{La question paysanne}

9 Face aux luttes politiques des femmes dans les grandes villes, fortement liées à une action syndicale au sein des usines, le contexte rural est tout différent. Malgré des conditions de vie elles aussi déplorables, la forte prégnance d'un patriarcat inspiré de l'autocratisme paternaliste des Tsars ainsi que l'influence de la religion contraignent les possibilités d'un soulèvement organisé. L'autocratie tsariste tout comme les troupes blanches contre-révolutionnaires ne cesseront de s'affirmer grâce au soutien de l'Église orthodoxe, elle-même structurée par la dévotion de nombreuses couches paysannes. Les bolcheviks comme les autres groupes révolutionnaires, jusqu'au libéraux du parti $\mathrm{KD}$, se heurteront constamment aux résistances paysannes et à une forme de conservatisme moral entretenu par le dogme religieux depuis des siècles. Cette réalité contraste avec les schèmes de représentation bolcheviques, essentiellement axés sur une ligne idéologique urbaine et prolétarienne, tandis que la Russie des Tsars est une société rurale et agricole. Marx, lui-même conscient de cette singularité, avait d'ailleurs été proche des lectures effectuées par les populistes russes (ancêtres des socialistesrévolutionnaires) à propos des collectivités rurales et des formes de protocommunisme qui y sont à l'œuvre. Son souci était de développer une analyse des rapports de production tels qu'ils existaient en Russie, de comprendre la singularité de son fonctionnement économique et social, non de calquer sur une réalité des schèmes de lecture induits par un contexte presque intégralement différent, celui du capitalisme anglais ou prussien. La posture bolchevique à propos de la question paysanne est complexe ; elle est à nouveau changeante au cours du temps. D'abord partisans d'une redistribution des terres au profit des muzhiki, comme l'atteste le slogan «pain, paix et terre ", les bolcheviks sont progressivement contraints par la Guerre civile d'instaurer un communisme de guerre où la collectivisation des campagnes sert le ravitaillement des villes et de l'armée. Ce moment cristallise une tension entre une réelle ouverture au monde rural et une première forme d'exploitation servile de la main d'œuvre agricole dans un contexte d'agitation et de terreur permanentes ${ }^{11}$. Cette exploitation sera radicalisée par les collectivisations meurtrières sous Staline à la suite du premier plan quinquennal de 1928-1929. La fin du communisme de guerre et du travail forcé cède le pas à la NEP en 1921, qui libéralise l'économie et rend possible l'émergence de nouvelles formes de propriété et de spéculation, notamment dans les campagnes. En s'inscrivant dans la lignée d'un Preobrajenski et de son capitalisme d'État fondé sur une accumulation «socialiste» primitive et une prolétarisation des campagnes, Staline scelle dans les années 1930 le destin funeste de nombreux paysans soumis au contrôle bureaucratisé du parti (grandes famines, dékoulakisation, purges, réquisitions forcées). Ce contexte rural hétérogène permet de comprendre les contradictions qui parcourent le rapport des bolcheviks avec la classe paysanne, fait de marginalisation, de paupérisation et de tentative de reconnaissance. 
10 Au sein de cette classe, le statut des femmes ne fait pas exception, il s'inscrit lui aussi dans des rapports de domination qui sont eux-mêmes le fruit de structures politiques et économiques propres. En un sens, la rencontre de conjonctures révolutionnaires n'a pas pris dans les campagnes : si des revendications et des agitations y ont bien eu lieu, elles sont bien souvent restées détachées de questions réellement politiques, au profit d'une urgence induite par la misère économique et les risques de famine. Pas de réelle rencontre donc dans ce cas entre des mouvements insurrectionnels et une éventuelle conscience politique au sein d'un prolétariat agricole non unifié. Celui-ci est traversé de contradictions que les bolcheviks ont souvent méconnues, contrairement à certains anarchistes et socialistes-révolutionnaires. En répondant à une conception téléologique de l'histoire - la révolution intégrale comme fin et par tous les moyens - le marxismeléninisme des bolcheviks n'a pas suffisamment pris en considération la nécessité de penser les conditions historiques d'une rencontre des conjonctures rurales et urbaines.

11 Les premières décisions politiques prises au lendemain de la révolution d'octobre 1917 à propos de la famille et des droits matrimoniaux reflètent une volonté de transformer profondément la condition des femmes, en occultant certes souvent une hétérogénéité sociale et idéologique, marquée par des résistances paysannes réelles.

\section{Les premières lois de reconnaissance politique et morale}

12 Les premiers décrets de décembre 1917 ainsi que le Premier code de la famille de 1918 apportent une réponse politique et juridique aux revendications révolutionnaires relatives aux droits des femmes: laïcisation du mariage, égalité absolue des époux, abolition de la différence entre enfants légitimes et illégitimes, et enfin réelle reconnaissance du droit au divorce. Le décret du 18 novembre 1920 vient compléter ces réformes par une légalisation de l'avortement thérapeutique gratuit ${ }^{12}$. Malgré l'officialisation d'un chamboulement moral, il ne faut pas nier les dissensions à l'intérieur même du groupe bolchevique. Ainsi, si Kollontaï représente une ligne plus anarchisante et individualiste, beaucoup défendent encore une forme de puritanisme moral, qui motivera plus tard une révision de ces réformes. Lénine, lui-même défenseur de l'émancipation des droits des femmes, a une position plus modérée, parfois ambiguë. Preuve de son opportunisme politique, le dirigeant bolchevique n'hésitera pas à enfermer la socialiste-révolutionnaire Maria Spiridonova pour hystérie en 1919. Victime d'une tentative d'assassinat orchestrée par Fanny Kaplan en 1918 au nom du socialisme, Lénine modifie constamment sa ligne idéologique au gré des évolutions politiques et n'hésite pas à réprimer les dissidences révolutionnaires (mencheviks, socialistes-révolutionnaires, anarchistes). Certains d'entre eux, parmi lesquels plusieurs femmes, seront enfermés dans le camp de rééducation par le travail des Solovki dès 1923. Staline accentuera ce processus en ciblant les membres (supposés) dissidents du PCUS.

13 L'une des idées fondatrices sous-tendant les mesures instaurées par les décrets consécutifs à la révolution (1917-1920) est la complète séparation entre l'Église et l'État. Dans l'héritage de la critique développée par Marx de la religion, plutôt adogmatique sur la question ${ }^{13}$, les révolutionnaires conçoivent l'institution religieuse comme une organisation et une reproduction de l'aliénation des classes dominées par la classe dominante, essentiellement masculine. En tant qu'elle est au service de l'autocratie 
monarchique de droit divin, la dévotion religieuse est comprise comme une forme de conscience fausse du monde. Elle est cette idéologie au service d'une docilité et d'une soumission, d'une reproduction de l'ordre politique, non d'une émancipation individuelle et collective. Face au dogme religieux, Marx et ses continuateurs souhaitent rendre aux sujets, et plus précisément aux femmes contraintes dans leur sexualité, dans leur morale et dans leurs gestes, une liberté absolue de conscience et d'action. Car c'est dans cette subjectivité libre que se situe pour Marx toute la question religieuse et toute l'importance d'un travail de critique sociale lucide et conscient. La séparation de l'Église et de l'État et la laïcisation des institutions constituent donc les fondements nécessaires à la libération idéologique, à la remise en cause de toute croyance, mais elles ne peuvent justifier dans les faits aucune répression des individualités, des croyants et des pratiquants (ce que privilégieront les bolcheviks), au risque de reconduire à la fois une répression politique sur des critères convictionnels et une stigmatisation des franges les plus marginales de la société, dont les paysannes.

Progressivement, hommes et femmes deviennent égaux face à la loi, ce que le Code des lois sur le mariage, la famille et la tutelle de 1926 tente d'instituer. Cependant, il ne faut pas occulter une réalité extrêmement brutale qui, en raison d'un contexte social, politique et économique précaire, tend à marginaliser les populations placées en situation de faiblesse économique. Principales victimes, les femmes et les enfants se voient confrontés à des "pères-fuyards » qui, une fois le divorce prononcé, changent de lieu de résidence et ne payent aucune pension alimentaire. Conjuguées à une croissance de la misère et des répressions au tournant des décennies 1920 et 1930, ces mesures ont pour conséquence une hausse du nombre d'enfants sans abri et une croissance de la prostitution. La lutte de l'anarchiste Evguénia Iaroslavskaïa-Markon est à ce titre emblématique. Se faisant le porte-voix du Lumpenproletariat marginalisé puis stigmatisé par le pouvoir soviétique ${ }^{14}$, ces petits criminels et ces prostituées errant dans les rues, elle est arrêtée puis envoyée au camp des Solovki où elle est exécutée en 1931. Ce moment marque le tournant ouvertement répressif d'un pouvoir empêtré dans des mesures dictatoriales et obnubilé par la collectivisation forcenée des campagnes et par l'industrialisation à outrance du territoire. Dans la foulée du plan quinquennal de 1928-1929, l'administration du GOULAG est créée en 1930. Elle enfermera indistinctement hommes et femmes supposés hostiles au pouvoir centralisé du Politburo.

\section{Le revirement conservateur sous Staline}

Si la révolution laissait entrevoir les possibilités de carrières politiques pour les femmes, le pouvoir strictement masculin qui s'instaure dans le courant des années 1930 autour des figures de Staline, Kaganovitch, Molotov, Vorochilov, Kalinine, et Khrouchtchev (auxquels on peut ajouter les successifs dirigeants du NKVD, Iagoda, Iejov et Béria) ne leur laisse presque aucune place. Plus encore, le Genotdel, section féminine du parti à la tête de laquelle on trouve un temps Kollontaï, est dissous en 1930 par Staline face aux critiques adressées par les femmes à l'encontre du Parti et de son impuissance à libérer le prolétariat féminin. La deuxième moitié des années 1930 est marquée par la Grande Terreur et les procès de Moscou, par la répression des socialistes et anarchistes du POUM lors de la Guerre d'Espagne et par un retour à un conservatisme moral justifié par l'urgence de la situation. Cette logique de l'urgence 
motive constamment le pouvoir dans l'application de ce qu'il considère être une dictature du prolétariat, selon l'acception de Marx. Or, face à des décisions absolument non communistes (accumulation primitive, libéralisation de l'économie après la NEP, exploitation productiviste des zeks du GOULAG, mise en concurrence des travailleurs détenus et des travailleurs « libres », punition sévère des grèves, des « vols de propriété collective", des "désertions", perpétuation des institutions que sont le salaire et l'argent, absence d'autonomie ouvrière quant à la gestion des moyens de production ${ }^{15}$ ), plusieurs commentateurs, dont l'anarchiste Alexandre Berkman ${ }^{16}$, ont interprété la surrépression stalinienne comme une dictature sur le prolétariat.

Plusieurs exemples de femmes victimes de cette répression, toutes arrêtées puis exécutées durant la Grande Terreur de 1937-1938, sont emblématiques de cette volonté d'éliminer les éléments les plus révolutionnaires. Evguénia Iouzefovna Belina, traductrice au journal La Tribune soviétique, avait été accusée d'espionnage; Anastasia Alekseïevna Bitsenko, socialiste-révolutionnaire ralliée aux bolcheviks, avait assassiné un général tsariste en 1906; Dorotea Wilhelmovna Fridlender, enseignante dans une école technique, était membre du Parti Communiste d'Allemagne; Anna Moisseïevna Bitter, professeure de géographie, était sans-parti mais fut réhabilitée après le $\mathrm{XX}^{\mathrm{e}}$ congrès du PCUS en 1956, comme la plupart des victimes communistes de la Grande Terreur.

17 Autre exemple significatif, celui de Marfa Ilinitchna Riazantesva, retraitée analphabète accusée d'appartenir à un groupe de religieux monarchistes contre-révolutionnaires. Cette dernière représente à elle seule tout l'échec du soviétisme stalinien. Incapable de proposer de réelle assistance publique pour les couches les plus dominées - petits paysans, petits délinquants, prostituées, sans-abri, garçons de ferme, retraités -, il s'empêtre paradoxalement dans une répression ayant tous les traits d'une marginalisation sociale fondée sur un hygiénisme. Tandis que le projet révolutionnaire initial était de supprimer les prisons comme institutions d'une justice de classe, le pouvoir stalinien radicalise une logique carcérale et pénitentiaire au nom de l'état d'exception.

En 1936, année des premiers procès de Moscou, un arrêté est prononcé : il interdit l'avortement et augmente les frais de divorce au nom de la protection de la famille et de la maternité. Face à ces mesures, de vives protestations s'expriment contre Staline lors de la consultation populaire. "Inculture populaire» selon lui. Seule exception : les femmes de détenus peuvent divorcer gratuitement, nouvelle preuve de l'instauration d'une double caste, d'une part celle des zeks reclus et marginaux et, d'autre part, celle de l'Homme soviétique nouveau. La « Grande Guerre patriotique » vient accentuer cette tendance. En 1944, un décret reconnait la nécessité d'un redressement démographique. La femme devient alors la mère nourricière des prolétaires, elle est selon les termes de Maria Markus un "instrument» au service de l'État ${ }^{17}$. La procédure de divorce est encore complexifiée juridiquement. Les hommes peuvent pour leur part engendrer des enfants naturels sans aucune responsabilité, les mères célibataires se voyant octroyer des aides d'État. Or, les mentalités ont considérablement évolué et les mœurs beaucoup plus libérales de la population soviétique entrent en contradiction avec cette législation de 1944, qui sera progressivement abandonnée après la mort de Staline en 1953. 


\section{Conclusions}

La lecture critique qui vient d'être ébauchée illustre la manière dont les premières tendances révolutionnaires, portées par des femmes dont l'action politique s'est affirmée dans la lutte, ont progressivement cédé la place à la réinstauration d'une véritable domination masculine en URSS, qui est le fruit d'une évolution de conjonctures historiques mais aussi d'un reniement progressif des idéaux révolutionnaire par le pouvoir masculin du PCUS des années 1930. De nombreuses femmes ont été victimes de la violence de ce système dans les camps du GOULAG, où les humiliations et les agressions sexuelles par les gardiens furent monnaie courante. Ces pratiques perpétuent une violence plus sous-terraine, symbolique, statutaire, sociale, en s'appliquant physiquement aux corps des femmes détenues. Considérées tantôt comme une marchandise sexuelle, tantôt comme des génitrices, tantôt comme des dépravées par le pouvoir stalinien, elles ont subi jusque dans l'intimité de leur corps un système d'exploitation proche des logiques productivistes du capitalisme privé, aux antipodes des projets d'émancipation des premières révolutionnaires. Dans le même temps, la marginalisation et la paupérisation de certaines femmes (en raison de la Guerre civile, des famines, de la guerre ou des répressions de masse) les ont contraintes à des formes de prostitution ou de survie à la limite de la légalité. Plus encore, ce pouvoir a accentué la répression de ces femmes devenues marginales, transformant le projet d'éducation, d'assistance et d'émancipation collective en une hygiénisation sociale.

Véra Figner, auteure des Mémoires d'une révolutionnaire ${ }^{18}$, par ailleurs traduite par Victor Serge, a écrit sur l'autoritarisme des Tsars, sur sa volonté de coupler une action politique révolutionnaire à un projet libertaire et antiautoritaire. Membre de Narodnaïa Volia et proche de Bakounine, elle fut enfermée plus de vingt ans par le pouvoir autocratique pour avoir participé à l'assassinat du Tsar Alexandre II. Attentive à la condition de la majorité des paysans et des paysannes russes, à leurs conceptions singulières de la liberté et de l'autonomie (Volia signifie à la fois liberté et volonté), elle défend l'idée d'une écoute attentive des minorités afin de penser l'action politique, à partir notamment des collectivités rurales. Critiquée sous le stalinisme sans pour autant être victime de la terreur, elle symbolise une nouvelle forme de singularité et d'hétérogénéité de pensée par rapport à la récurrence d'un modèle masculin fait d'exploitation et de domination. La lutte organisée par les femmes révolutionnaires en Russie, si elle s'est constamment heurtée à des obstacles patriarcaux, a tenté de montrer la nécessité d'une convergence des combats politiques où la lutte pour la reconnaissance des droits sociaux, politiques, économiques et de genre se pensent de façon indissociable. En un sens ce matérialisme de la rencontre définit les conditions de possibilité d'une émancipation intégrale des femmes conjointement à une émancipation économique et politique du prolétariat et de chaque groupe rendu subalterne. Il n'offre aucune clef de lecture prédéfinie, aucune téléologie ni aucune eschatologie, mais il invite au contraire à penser la conjoncture comme le lieu même de naissance du projet politique révolutionnaire. 


\section{NOTES}

1. Je tiens à remercier tout particulièrement Julie Ricard des Territoires de la Mémoire qui, dans le cadre de l'exposition Goulag. Visages et rouages d'une répression organisée à La Cité Miroir de mars à août 2020, a soumis l'idée d'une visite orientée sur la question des droits des femmes en collaboration avec le Collectif contre les violences familiales. Cet article est le fruit de réflexions et de discussions développées en amont et en aval de cette initiative. Voir également à ce propos Thomas Franck, Dictature et Prolétariat. De l'autocratie tsariste au dirigisme bolchevique, Liège, MNEMA Éditions, coll. «Les Cahiers du Centre Pluridisciplinaire de la Transmission de la Mémoire ", 2020.

2. Fabio Bruschi, Le Matérialisme politique de Louis Althusser, Sesto San Giovanni, Mimésis, 2020.

3. Friedrich Engels, Origine de la famille, de la propriété privée et de l'État, Paris, Éditions sociales, 1972.

4. Alexandra Kollontaï, Le Communisme et la famille, citée in Kendall E. Bailes et Marie-José Imbert, "Alexandra Kollontaï et la Nouvelle Morale ", in Cahiers du monde russe et soviétique, Vol 6, $\mathrm{N}^{\circ} 4$, 1965, p. 476.

5. Alexandra Kollontaï, La base sociale de la question féminine, 1909, in Marxists.org, URL : https:// www.marxists.org/francais/kollontai/works/1909/00/bases_sociales.htm.

6. Rosa Luxembourg, Suffrage féminin et lutte de classes, mai 1912, in Marxists.org, URL : https:// www.marxists.org/francais/luxembur/works/1912/05/suffrage.htm.

7. Dans la section 6 de son Cahier 23, Antonio Gramsci établit un rapport entre la nécessité de l'émergence d'une nouvelle culture et l'hégémonie revendiquée par les groupes subalternes. Si cette réflexion fourmille dans l'ensemble de son œuvre, on citera ici un passage qui nous paraît important en ce qu'il met en évidence la nécessité d'un travail collectif (culturel) préalable à la valorisation du travail (artistique) individuel : «Qu'il faille parler, pour être exact, de lutte pour une "nouvelle culture" et non pour un "nouvel art" (au sens immédiat), cela semble évident. Peut-être ne peut-on même pas dire, pour être exact, qu'on lutte pour un nouveau contenu de l'art, parce que celui-ci ne peut être pensé abstraitement, séparément de la forme. Lutter pour un nouvel art signifierait lutter pour créer de nouveaux artistes individuels, ce qui est absurde car on ne peut créer artificiellement les artistes. On doit parler de lutte pour une nouvelle culture, c'est-à-dire pour une nouvelle vie morale qui ne peut pas ne pas être intimement liée à une nouvelle intuition de la vie, jusqu'à ce qu'elle devienne une nouvelle façon de sentir et de voir la réalité et donc un monde intimement lié aux "artistes possibles" et aux "œuvres d'art possibles". Qu'on ne puisse créer artificiellement des artistes individuels ne signifie donc pas que le nouveau monde culturel, pour lequel on lutte, en suscitant passions et chaleureuse humanité, ne suscite pas nécessairement de "nouveaux artistes" ; c'est-à-dire qu'on ne peut pas affirmer que Pierre et Paul deviendront des artistes, mais qu'on peut affirmer que du mouvement naîtront de nouveaux artistes. Un nouveau groupe social qui entre dans la vie de l'histoire avec un comportement hégémonique, une confiance en soi qu'il n'avait pas auparavant, ne peut pas ne pas faire surgir de ses profondeurs des personnalités qui, précédemment, n'auraient pas trouvé une force suffisante pour s'exprimer en une certaine direction de façon complète " (Antonio Gramsci, Cahiers de prison. Cahiers 19, 20, 21, 22, 23, 24, 25, 26, 27, 28 et 29, Paris, Gallimard, coll. "Bibliothèque de Philosophie ", 1991, p. 225-226).

8. Antonio Gramsci, Cahiers de prison. Cahiers 6, 7 ,8 et 9, Paris, Gallimard, coll. «Bibliothèque de Philosophie ", 1983, p. 217.

9. La chose est très bien analysée par Pierre Broué dans Le Parti bolchevique, Paris, Minuit, 1963 et par Orlando Figes dans son Histoire de la révolution russe, Paris, Gallimard, 2009. Le gouvernement 
provisoire de Kerenski tente une nouvelle fois de placer le parti bolchevique dans l'illégalité en l'interdisant en juillet 1917.

10. Pierre Broué, «Remarques sur l'histoire du parti bolchevik », in Arguments, $n^{\circ} 25-26,1^{\text {er }}$ et $2^{\text {ème }}$ trimestres 1962, p. 57.

11. Les pogromes et les massacres commis dans les campagnes par les armées de Denikine, de Koltchak, de Kornilov et, plus généralement, par les troupes blanches sont au fondement de ce contexte de terreur et d'escalade de violence. La répression vise précisément des ouvriers, des juifs et des militants révolutionnaires, suivant un amalgame « judéo-bolchevique » volontaire.

12. Helène Yvert-Jalu, «L'histoire du divorce en Russie soviétique. Ses rapports avec la politique familiale et les réalités sociales ", in Population, Vol 23, N¹, 1981, p. 41-61.

13. Voir notamment Karl Marx, Critique de la philosophie du droit de Hegel, Paris, Allia, 1998 et Karl Marx et Friedrich Engels, Sur la Religion. Textes choisis, Paris, Editions sociales, 1960.

14. Evguénia Iaroslavskaïa-Markon, Révoltée, Paris, Seuil, 2017.

15. Voir à ce propos Maximilien Rubel, Marx critique du marxisme, Paris, Payot, 1974.

16. Alexandre Berkman, Le Mythe bolchevik. Journal 1920-1922, Paris, Klincksieck, coll. «Critique de la politique », 2017.

17. Maria Markus, "L'émancipation des femmes dans l'impasse ", in Impact, Science et Société, citée in Yvert-Jalu, art. cit.

18. Véra Figner, Mémoires d'une révolutionnaire. Le temps retrouvé, Paris, Gallimard, 2017.

\section{ABSTRACTS}

Cet article étudie les évolutions conjoncturelles en Union Soviétique en lien avec la place prise par les femmes dans les mouvements révolutionnaires pour une reconnaissance de leurs droits, de leur autonomie politique et de leurs libertés morales. Dans un premier temps, il met au jour le renversement du patriarcat des Tsars par l'instauration, d'une part, d'un pouvoir du prolétariat féminin et, d'autre part, d'une reconnaissance juridique (divorce, avortement, égalité juridique et politique). Dans un second temps, il s'attarde sur le revirement conservateur insufflé sous Staline et la manière dont le Politburo réinstaure une domination masculine allant à l'encontre des acquis de la révolution. Cette recherche vise à comprendre la manière dont une convergence des luttes sociales, politiques et de genre a amené à des réformes profondes de la condition du prolétariat féminin. Elle montre également en quoi les structures politiques fondées sur une domination et une exploitation (tant sous le tsarisme que sous le stalinisme redéfini comme capitalisme d'État) vont constamment dans le sens d'une mise à mal des luttes et des acquis de ce même prolétariat.

\section{INDEX}

Chronological index: $1917-1953$

Geographical index: URSS

Mots-clés: prolétariat féminin, matérialisme de la rencontre, URSS, droits des femmes, révolution de février, révolution d'octobre, autonomie politique, domination masculine, patriarcat 


\section{AUTHOR}

\section{THOMAS FRANCK}

Chargé de recherche à La Cité Miroir et collaborateur scientifique à l'Université de Liège, membre du GRM 